\title{
Characterization and analysis of a transcriptome from the boreal spider
} crab Hyas araneus

\author{
Q1 Lars Harms a,*, Stephan Frickenhaus b, Melanie Schiffer ${ }^{\text {a }}$, Felix C. Mark a , Daniela Storch a , Hans-Otto Pörtner a , \\ Christoph Held ${ }^{\mathrm{c}}$, Magnus Lucassen ${ }^{\mathrm{a}}$ \\ a Integrative Ecophysiology, Alfred Wegener Institute, Helmholtz Centre for Polar and Marine Research, Bremerhaven, Germany \\ b Scientific Computing, Alfred Wegener Institute, Helmholtz Centre for Polar and Marine Research, Bremerhaven, Germany \\ c Functional Ecology, Alfred Wegener Institute, Helmholtz Centre for Polar and Marine Research, Bremerhaven, Germany
}

\section{A R T I C L E I N F O}

\section{Article history:}

Received 22 May 2013

Received in revised form 5 September 2013

Accepted 30 September 2013

Available online $\mathrm{xxxx}$

\section{Keywords:}

Spider crab (Hyas araneus)

Crustacea

Transcriptome

Illumina sequencing

454 sequencing

Markov Clustering Algorithm

Anti-viral immunity

Reverse transcriptase

\begin{abstract}
A B S T R A C T
Research investigating the genetic basis of physiological responses has significantly broadened our understand- 27 ing of the mechanisms underlying organismic response to environmental change. However, genomic data are 28 currently available for few taxa only, thus excluding physiological model species from this approach. In this 29 study we report the transcriptome of the model organism Hyas araneus from Spitsbergen (Arctic). We generated 30 20,479 transcripts, using the 454 GS FLX sequencing technology in combination with an Illumina HiSeq sequenc- 31 ing approach. Annotation by Blastx revealed 7159 blast hits in the NCBI non-redundant protein database. The 32 Q4 comparison between the spider crab H. araneus transcriptome and EST libraries of the European lobster Homarus 33 americanus and the porcelain crab Petrolisthes cinctipes yielded 3229/2581 sequences with a significant hit, 34 respectively. The clustering by the Markov Clustering Algorithm (MCL) revealed a common core of 1710 clusters 35 present in all three species and 5903 unique clusters for $H$. araneus. The combined sequencing approaches 36 generated transcripts that will greatly expand the limited genomic data available for crustaceans. We introduce 37 the MCL clustering for transcriptome comparisons as a simple approach to estimate similarities between 38 transcriptomic libraries of different size and quality and to analyze homologies within the selected group of 39 species. In particular, we identified a large variety of reverse transcriptase (RT) sequences not only in the 40 Q3 $H$. araneus transcriptome and other decapod crustaceans, but also sea urchin, supporting the hypothesis of a herita- 41 ble, anti-viral immunity and the proposed viral fragment integration by host-derived RTs in marine invertebrates. 42
\end{abstract} (C) 2013 Published by Elsevier Inc. 43

\section{Introduction}

The great spider crab, Hyas araneus, is a benthic decapod crab that lives on sublitoral rocky or sandy substrates to a depth of $50 \mathrm{~m}$ (Hayward and Ryland, 1990). Within the North-East Atlantic region it is distributed along a latitudinal gradient from the English Channel up to the Arctic regions of Spitsbergen, where it represents one of the most prominent brachyuran crabs (Zittier et al., 2012). The size of its distribution range and the corresponding cline in environmental conditions make $H$. araneus an ideal species to study the effects of environmental changes as well as functional differentiation between populations. For example, decreased larval developmental rates in Arctic compared to temperate populations suggest adaptation to the polar cold (Walther et al., 2010). Elevated seawater $\mathrm{PCO}_{2}$ (as projected by ocean acidification scenarios) caused an increase in metabolic

Abbreviations: MCL, Markov Clustering Algorithm; CEGMA, Core Eukaryotic Genes Mapping Approach.

* Corresponding author.

E-mail address: LHarms@gmx.net (L. Harms). rate during larval development pointing to higher metabolic costs in 62 larvae (Schiffer et al., 2012). Adult $H$. araneus displayed increased heat 63 sensitivity under elevated $\mathrm{CO}_{2}$ levels with potential consequences for 64 biogeographical boundaries (Walther et al., 2009). However, the genetic 65 basis of these responses to environmental changes has so far only been 66 investigated for a limited number of candidate genes. For example, 67 hyastatin, a peptide involved in haemolymph antimicrobial defense, 68 has been isolated, and the importance of the cys-containing region for 69 the antimicrobial activity and a possible multifunctional character has 70 been demonstrated (Sperstad et al., 2009). The reason for the small 71 number of studies is likely the lack of genomic information in databases 72 like the National Center for Biotechnology Information (NCBI). Current- 73 ly, only 26 nucleotide sequences of $H$. araneus are published in NCBI. $\quad 74$

In recent years, Next Generation Sequencing (NGS) has made it pos- 75 sible to approach this problem by sequencing and assembly of entire ge- 76 nomes of ecologically relevant species (for review see Wheat, 2010). 77 However, for non-model organisms, sequencing a transcriptome rather 78 than the genome to obtain the genetic data is advantageous for many 79 reasons. The generation of sequence data is quick, it is relatively cost- 80 effective and can thus provide the genetic basis for studies with 81 fewer resources. Further, transcriptome sequencing can provide both 82 
expression and coding data, using RNA-seq (Martin and Wang, 2011). Using different tissues and differentially treated animals it is possible to capture variations in coding sequences, stress induced sequences as well as differences in the expression level. Respective approaches have already been applied to a number of marine invertebrates to achieve insights into expression information (Giant Ezo scallop (Hou et al., 2011); common octopus (Zhang et al., 2012); 2 Mollusca, 2 Arthropoda, 2 Annelida, 2 Memertea, 2 Porifera (Riesgo et al., 2012); pearl oyster (Shi et al., 2013)) thereby expanding the existing genetic resources massively.

Thus, the objective of the present study was to fundamentally characterize the transcriptome of $H$. araneus. For analyzing specific homologies within decapod transcriptomes and for identifying common and specific gene clusters of the selected group of species we introduced the Markov Clustering Algorithm (MCL) clustering approach.

To develop an extensive transcriptome of $H$. araneus we combined the 454 and Illumina sequencing technologies on normalized and common cDNA libraries constructed from pooled samples of multiple tissues from animals treated with different environmental conditions (see Materials and methods). We assembled the sequences to reconstruct transcripts potentially representing the $H$. araneus transcriptome. Because no reference genome is available for $H$. araneus we assembled the transcriptome de novo. There are several de novo tools available, but none represent the perfect solution (Kumar and Blaxter, 2010). To obtain a comprehensive and high-quality de novo assembly of the $H$. araneus transcriptome, we tested different assembling tools and compared the resulting assemblies. In the second part we analyzed the functionally annotated transcriptome for particular features and compared the identified sequences with available sequence information of other decapod crustaceans using the MCL-clustering to reveal homologies within the selected group of species.

The approach illustrates a potential methodological framework and may promote further transcriptome studies in non-model organisms. The transcriptome obtained for $H$. araneus will become essential for future analyses and annotations and also provide useful information for future functional genomic studies in crustaceans.

\section{Materials and methods}

\subsection{Sample preparation and RNA extraction}

Adult specimens of the boreal spider crab $H$. araneus were collected in the Kongsfjord ( $\mathrm{N} 78^{\circ} 58.635^{\prime}$; E $\left.11^{\circ} 29.454^{\prime}\right)$ at the west coast of Spitsbergen (Norway). Animals were acclimated for 10 weeks in flow through aquaria systems to 6 different treatments of 3 seawater $P \mathrm{CO}_{2}$ values $(390,1120$ and $1960 \mu \mathrm{atm})$ combined with two temperatures (5 and $10{ }^{\circ} \mathrm{C}$ ), respectively. Tissue samples comprising of all 6 gillarches, tegument, heart, hepatopancreas, testis and pincer muscle were collected from four to six animals per treatment and directly frozen in liquid nitrogen. Samples were stored at $-80{ }^{\circ} \mathrm{C}$ until used for RNA extraction. Total tissue RNA was extracted by using the RNeasy Mini Kit according to the "Purification of Total RNA from Animal Tissue" protocol (QIAGEN, Hilden, Germany). RNA quantities were determined by a NanoDrop 2000c spectrometer (PeqLab, Erlangen, Germany), and RNA was analyzed for quality by microfluidic electrophoresis in an Agilent 2100 Bioanalyzer (Agilent Technologies).

\subsection{Sequencing and assembly}

To generate the transcriptome of the non-model organism $H$. araneus, two different sequencing approaches were used. First, a 454 pyrosequencing approach based on normalized cDNA libraries was applied, serving as a basis for the assembly. Using samples from multiple tissues and differentially treated animals (rearing temperature and $\mathrm{PCO}_{2}$ level) as well as using a normalized cDNA libraries allow for a comprehensive transcriptome, capturing variations in coding sequences, stress induced sequences as well as low expressed genes. Two separate cDNA libraries 144 were sequenced by 454: a library exclusively based on gill samples and a 145 library based on samples of a mixture of tissues. For the H. araneus gill li- 146 brary, the same amount of RNA was collected from each gill of 4 animals 147 per treatment and pooled in one sample. The same was done for all other 148 tissues to prepare the material for a mixed tissue library. Both mixtures 149 were used for the library constructions by the Max Planck Institute for 150 Molecular Genetics (Berlin, Germany). Total RNA of the two pools (gill 151 and mixed tissue) was used for CDNA synthesis using the SMART proto- 152 col (Mint-Universal cDNA synthesis kit, Evrogen, Moscow, Russia). The 153 cDNA was subsequently normalized using duplex-specific nuclease 154 and re-amplified thereafter following the instructions of the "Trimmer 155 Kit" (Evrogen, Moscow, Russia). Sequencing libraries were prepared 156 from cDNA using the "GS FLX Titanium General Library Preparation 157 Kit" (Roche, Basel, Switzerland). Before sequencing, the libraries were 158 amplified by polymerase chain reaction (PCR) using the 'GS FLX Titani- 159 um LV emPCR Kit' (Roche, Basel, Switzerland) (De Gregoris et al., 160 2011). Sequencing was performed by the Max Planck Institute for 161 Molecular Genetics (Berlin, Germany) on a 454 Genome Sequencer 162 FLX using the Titanium chemistry (Roche). Initial quality control and 163 filtering of adapters and barcodes was performed at the Max Planck In- 164 stitute for Molecular Genetics (Berlin, Germany). Both cleaned libraries 165 were combined for the subsequent de novo assembly. To optimize the 166 quality of the de novo transcriptome assembly, we compared two differ- 167 ent assembler programs: GS De Novo Assembler version 2.6 (Newbler, 168 Roche) and MIRA 3.0 (Chevreux and Wetter, 1999). We tested each pro- Q7 gram with the following main assembly parameters: minimum percent- 170 age identities of 95\%, and minimum overlap length of $40 \mathrm{bp}$ for MIRA, 171 and 40 bp for the GS De Novo Assembler. The "-cdna" mode was used 172 for the GS De Novo Assembler. The final de novo assembly by GS De 173 Novo Assembler was chosen based on basic assembly metrics and 174 performance in terms of completeness and contiguity. 175

Secondly, an Illumina sequencing approach was used to enhance the 176 454 based transcriptome. Six different cDNA libraries based on samples 177 of the six different treatments were sequenced. For each treatment, total 178 RNA from all gills of 4 animals was pooled and used for the library con- 179 struction by GATC Biotech (Konstanz, Germany). Libraries for each treat- 180 ment were constructed according to the 'SMART protocol for Illumina 181 sequencing' (Clontech, Mountain View, CA, USA). Illumina single-end 182 sequencing was performed on a HiSeq 2000 Sequencer by GATC Biotech. 183 Initial quality control and filtering of adapters was performed by GATC 184 Biotech. In addition, obtained raw reads were quality controlled by 185 FastQC (Babraham Institute, Cambridge, UK) and cleaned using the 186 FastX-Toolkit (Hannon Lab - Cold Spring Harbor Laboratory, NY, 187 USA). Quality control was performed using the following parameters: 188 minimum quality score of 20, minimum percentage of bases within 189 the quality score of 90 and a minimum length of 25 bases. To enhance 190 the set of GS De Novo Assembler-assembled contigs, obtained 191 Illumina-data from the six libraries were combined and reads were as- 192 sembled de novo with ABySS version 1.3.2 (Simpson et al., 2009) with 193 $\mathrm{k}=26$, minimum overlap length of $30 \mathrm{bp}$ and minimum sequence 194 identity of 0.9. Considerable overlaps with the GS De Novo Assembler- 195 assembled 454-data were detected with blastn (word size 8), removing 196 Abyss-contigs above E-value $10^{-10}$ and length below $500 \mathrm{bp}$. The 197 transcriptome of $H$. araneus was deposited in the 'European Nucleo- 198 tide Archive' (ENA) at the 'European Molecular Biological Laborato- 199 ry-European Bioinformatics Institute' (EMBL-EBI) (Accession range: 200 HAAI01000001-HAAI01019199).

\subsection{Functional annotation}

Functional annotation of the $H$. araneus transcriptome was accom- 203 plished using the Blast2GO software v.2.6.0 (Conesa et al., 2005; Gotz 204 et al., 2008). Homology searches were performed using Blastx against 205 the NCBI non-redundant protein database. Blast searches were per- 206 formed with an E-value cut-off of $1 \mathrm{E}^{-3}$. For the Gene Ontology (GO) 207 
classification of the blasthits, the default parameters were used (E-value $<1 \mathrm{E}^{-6}$, annotation cut-off $>55$ and a GO weight $>5$ ). The annotated most specific GO terms were traced back to the second level parent term using the R Bioconductor package "GO.db" (Carson et al., 2010).

\subsection{Comparative analysis}

The assembled transcript sequences were compared with EST sequence libraries from Homarus americanus and Petrolisthes cinctipes obtained from Genbank. After filtering for length $>500$, a set of 25,185, 75,208 and 13,706 sequences (from $H$. americanus, $P$. cinctipes, and $H$. araneus, respectively) were clustered following the Markov Cluster Algorithm (MCL) (Enright et al., 2002) based on tBlastx tables (all against all) with an $\mathrm{E}$-value $<10^{-9}$ and negative $\log _{10}$ E-value as similarity. The MCL-inflation parameter was $I=2$. The obtained set of 35,440 MCL-clusters was divided in species-specific or overlapping groups. Library-specific sequence counts within these cluster sets were computed. All analyses were performed in R (R Core Team, 2012). The R-script producing the counts in Venn-diagrams for clusters and sequences is available on our web-server http://www.awi.de/en/go/bioinformatics. The common core of clustered transcript sequences comprises 3245 $H$. araneus sequences of which a total of 2194 were found annotated in Blast2GO. This set has been taken for a GO enrichment analysis against the full set of annotated $H$. araneus transcript sequences (Fisher's exact test). The dataset of CEGMA 2.4 (Parra et al., 2007) was used to screen transcript sequences longer than 200 bp for universal eukaryotic functions using trpsblastn (Altschul et al., 1997) applied as in Windisch et al. (2012). Top CEGMA hits were analyzed on the basis of MCL clustering results for the core set of $H$. araneus sequences and the $H$. araneus specific MCL clusters.

\subsection{Comparative analysis of reverse transcriptase sequences}

For an extended analysis resolving similarity features with reverse transcriptase (RT) sequences, tBlastx analyses of published transcript sequences from Drosophila melanogaster $(\mathrm{N}=27,539)$ and Strongylocentrotus purpuratus $(\mathrm{N}=23,057)$ were incorporated in MCL with a more stringent lower E-value cut-off $1 \mathrm{E}^{-25}$. For this, $H$. araneus sequences were pooled with the $H$. americanus and $P$. cinctipes sequence libraries as crustaceans. Sequence IDs of all non- $H$. araneus libraries used in the tBlastx runs are listed in the supplement text file contained in Xseq-IDs.zip.

\section{Results and discussion}

\subsection{4-sequencing and assembly}

The two 454 pyro-sequencing runs based on normalized cDNA libraries constructed with total RNA from 6 different tissues (gills, tegument, heart, hepatopancreas, testis and pincer muscle) yielded a total of 1,111,880 reads with $335 \mathrm{Mbp}$ and an average length of $550 \mathrm{bp}$ (Table 1). The 454 reads originating from the two sequenced cDNA libraries were assembled with GS De Novo Assembler 2.6 (Newbler, Roche). After internal trimming, a total of 824,230 reads (260 Mbp) with an average length of 300 bp were assembled into 16,614 isotigs. The size of the reads extends to a maximum of $871 \mathrm{bp}$ with a peak between 300 and $480 \mathrm{bp}$ (Fig. 1A). The obtained isotigs had a maximum length of $6697 \mathrm{bp}$, an average length of $668 \mathrm{bp}$ and a N50 isotig size of 751 bp (Table 1 ). Isotigs with a length shorter than 100 bp were excluded from the analysis. The size distribution of the isotigs ranges from 100 to $6697 \mathrm{bp}$ with a peak between 400 and $600 \mathrm{bp}$ (Fig. 1B). The estimated average fold coverage of the isotigs was 6 and ranged from 1 to more than 2000 (Fig. 2).
Table 1

t1.1

454 sequence and assembly statistics. Gill and mixed tissue sequencings are combined for $t 1.2$ statistics. Only isotigs with a length greater than $100 \mathrm{bp}$ are considered in the assembly t1.3 statistics.

\begin{tabular}{l}
\hline Raw sequencing reads \\
Number of reads (gill tissue) \\
Number of reads (mixed tissue) \\
Number of reads (total) \\
Total size (bp) \\
Average size (bp) \\
Aligned reads \\
Number of reads \\
Total size (bp) \\
Average size (bp) \\
Assembly statistics \\
Number of isotigs \\
Total size (bp) \\
Average size (bp) \\
Maximum length, bp
\end{tabular}

\subsection{Enhancement of the transcriptome by Illumina sequencing}

An Illumina sequencing approach was carried out with total RNA 265 from gill tissue generated from animals long-term exposed to six differ- 266 ent climate conditions. The obtained sequences were used to enhance 267 the transcriptome generated by the 454 de novo assembly. The Illumina 268 sequencing runs yielded a total of 98,508,658 reads with $9457 \mathrm{Mbp}$ and 269 an average length of $96 \mathrm{bp}$ (Table 2). The Illumina reads originating from 270 the six treatments were assembled into contigs by use of the ABySS 271 Assembler (Simpson et al., 2009). A total of 55,354,912 reads with an 272

A

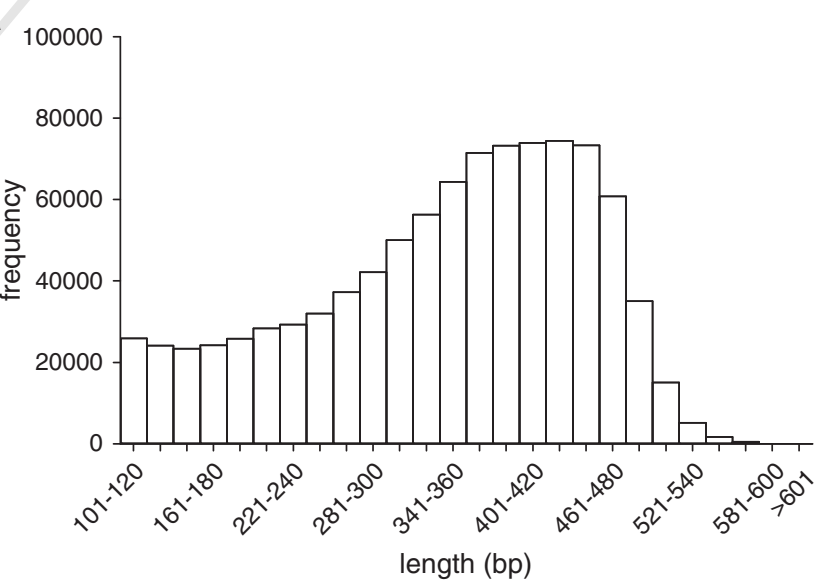

B

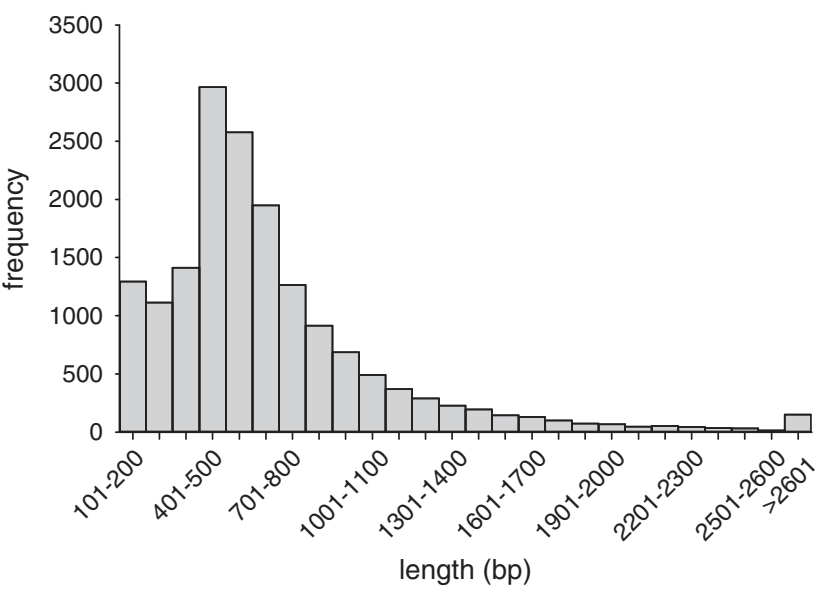

Fig. 1. Size distribution of reads and isotigs from 454 pyrosequencing. Reads (A) and isotigs (B) longer than $100 \mathrm{bp}$ are considered. 


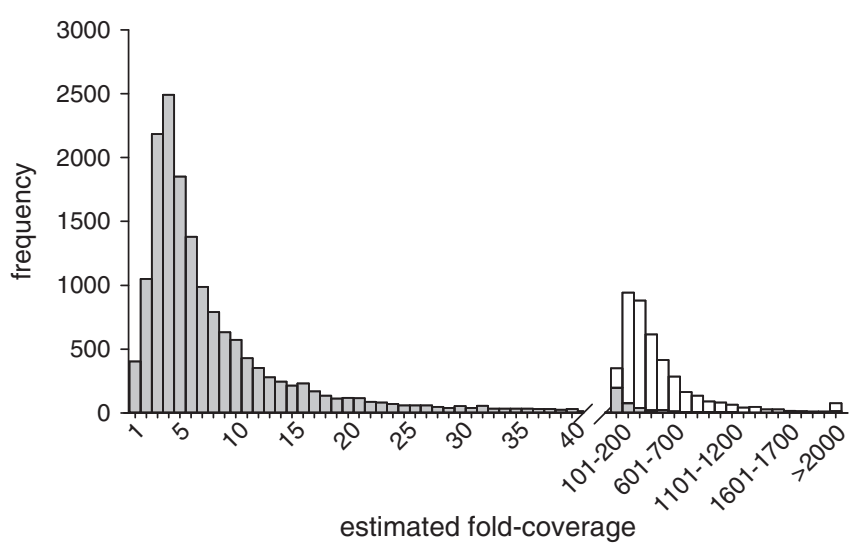

Fig. 2. Estimated fold-coverage of assembled isotigs/contigs and frequency of sequences with an according fold-coverage. Isotigs from 454 assembly (gray) and contigs from Illumina assembly (white).

average length of 61 bp were assembled into 175,612 contigs with a length greater than $100 \mathrm{bp}$. The contigs of the Illumina assembly had a maximum length of $3094 \mathrm{bp}$ and an average length of $195 \mathrm{bp}$ and a N50 isotig size of $213 \mathrm{bp}$ (Table 2). A relatively short average contig length of only $195 \mathrm{bp}$ can be explained by the short reads of the Illumina sequencing (61 bp) and the lack of a reference transcriptome/genome in non-model organisms. Similar results were reported for the non-model organism Radix balthica (snail) testing four different assemblers (Feldmeyer et al., 2011). We focused on transcripts of potentially greater functional relevance and excluded contigs with a length shorter than 500 bp from the ABySS Illumina assembly. All Illumina-based contigs showing an overlap with the Newbler 454 contigs were excluded to avoid redundancy. In total, 3865 contigs were used for further analysis and added to the existing 454 assembly to complement the transcriptome to a total of 20,479 transcript sequences. The estimated average fold coverage of the reduced set of contigs was 471 and ranged from 100 to over 2000, whereas - due to the larger sequence volume - the contigs led to a distinctly greater coverage than the isotigs of the 454 assembly (Fig. 2).

Recently, a comparative description of ten invertebrate transcriptomes was based solely on Illumina de novo sequencing and assembly (Riesgo et al., 2012), emphasizing the importance of sequencing invertebrate non-model species as a powerful basis for phylogenetic and functional genomic studies. In average, about $40 \%$ of all reads could be

Table 2

Illumina sequence and assembly statistics. Sequencings of treatments are combined for statistics. Only contigs with a length greater than $100 \mathrm{bp}$ are considered in the Illumina sequence statistics.

\begin{tabular}{ll}
\hline Raw sequencing reads & \\
Number of reads (treatment 1) & $12,670,506$ \\
Number of reads (treatment 2) & $18,137,025$ \\
Number of reads (treatment 3) & $12,363,308$ \\
Number of reads (treatment 4) & $21,113,665$ \\
Number of reads (treatment 5) & $21,110,842$ \\
Number of reads (treatment 6) & $13,113,312$ \\
Number of reads (total) & $98,508,658$ \\
Total size (bp) & $9,456,831,168$ \\
Average size (bp) & 96 \\
Aligned reads & \\
Number of reads & $55,354,912$ \\
Total size (bp) & $3,397,642,905$ \\
Average size (bp) & 61 \\
Assembly statistics & \\
Number of contigs & 175,612 \\
Total size (bp) & $34,271,175$ \\
Average size (bp) & 195 \\
Maximum length (bp) & 3094 \\
\hline
\end{tabular}

assembled, resulting in about 67,000 to 210,000 contigs across the ten 297 species (Riesgo et al., 2012). Based on our Illumina approach, we were 298 able to assemble slightly more reads (56\%) into a comparable number 299 of contigs within the present sequencing project.

\subsection{Functional analysis}

301

In order to annotate the consensus sequences, a Blastx search against 302 the NCBI non-redundant (nr) protein database was performed using the 303 Blast2GO suite (Conesa et al., 2005; Gotz et al., 2008). For the 20,479 304 transcripts the search revealed 7159 (35\%) significant blast hits $\left(1 \mathrm{E}^{-3} 305\right.$ cutoff threshold), which corresponded to 5962 unique accession num- 306 bers. The large number of transcripts without a significant blast hit 307 (65\%) is probably caused by a high proportion of novel genes and the 308 lack of fully annotated transcriptomes in closely related crustaceans. 309 The distribution of annotated and non-annotated transcripts is only 310 slightly influenced by the length, which can be explained by the fact 311 that a moderately restrictive E-value cut-off was used to obtain a com- 312 prehensive set of blasthits. A similar ratio of annotated and non- 313 annotated isotigs/contigs was observed in the transcriptome (Fig. 3). 314 In contrast, the quality of the annotation strongly depended on the tran- 315 script lengths. Table 3 lists the 20 consensus sequences with the highest 316 E-value and the highest score. All transcripts with a strong match in the 317 blast search belonged to sequences with a great length (>1500 bp). 318 Unsurprisingly, there are several heat-shock proteins included in the 319 top 20 list, as heat-shock proteins are often conserved across phyla 320 (Lindquist and Craig, 1988). Furthermore, a potential bias due to the 321 large number of studies with a focus on specific gene groups must 322 certainly be taken into account.

Gene Ontology (GO) terms of the $H$. araneus transcriptome were 324 analyzed using Blast2GO (Consortium, 2008). Blast2GO provides infor- 325 mation on the 'Molecular Function', the 'Cellular Component' and the 326 'Biological Process' for each sequence. In total, 27,074 GO terms could 327 be allocated for 4156 (58.1\%) sequences. The annotated GO terms are 328 grouped in 7226 (26.69\%) on 'Molecular Function', in 6414 (23.69\%) 329 on 'Cellular Component' and in 13,434 (49.62\%) on 'Biological Process' 330 (Fig. 4). For each sequence, the specific annotated GO term was mapped 331 to the second level parent term to obtain a broader overview of the 332 functionally grouped transcripts for the three GO ontologies (Fig. 4). 333 The hierarchical order of the GO allows to consider gene sets involved 334 in a specific process at a specific detail level of interest. For the 'Biologi- 335 cal Processes', the most frequent categories were 'cellular process' 336 (28.7\%), 'biological regulation' (23.3\%), 'cellular component organization 337 or biogenesis' (13.5\%) and 'developmental process' (13.1\%), followed by 338 'response to stimulus' (6.5\%), 'establishment of localization' (6.4\%), and 339

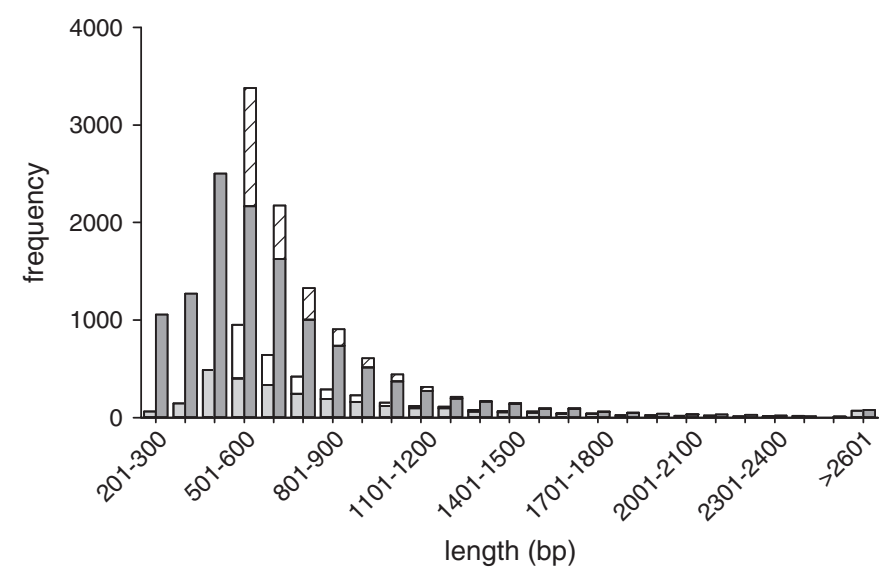

Fig. 3. Size distribution of annotated and non-annotated isotigs ( 454 sequencing)/contigs (Illumina sequencing). Annotated isotigs (gray), annotated contigs (white), non-annotated isotigs (dark gray) and non-annotated contigs (white with diagonal lines). 
Table 3

Top 20 high quality annotations of the Hyas araneus transcriptome. Sequences with highest score in Blastx search.

\begin{tabular}{|c|c|c|c|c|c|}
\hline Putative sequence description & Length & Score & ACC number & Species & Type \\
\hline Myosin heavy chain type a & 6490 & 2510 & BAK61429.1 & Marsupenaeus japonicus & Full length \\
\hline Elongation factor 2 & 3273 & 1556 & ACS36538.1 & Homarus americanus & Full length \\
\hline $\mathrm{Na}^{+} / \mathrm{K}^{+}$ATPase alpha subunit & 4496 & 1427 & AAG47843.1 & Callinectes sapidus & Partial \\
\hline Myosin heavy chain type b & 5507 & 1413 & BAK61430.1 & Marsupenaeus japonicus & Full length \\
\hline Low-density lipoprotein receptor protein like & 2477 & 1161 & XP_002430267.1 & Pediculus humanus corporis & Partial \\
\hline UDP-n-acetylglucosamine, n-acetylglucosaminyltransferase & 2180 & 1153 & XP_003249419.1 & Apis mellifera & Partial \\
\hline Heat shock protein 70 & 2427 & 1046 & CAL68989.1 & Cyanagraea praedator & Full length \\
\hline Heat shock protein 70 & 2204 & 1046 & ACE79213.1 & Scylla paramamosain & Full length \\
\hline hypothetical protein & 3414 & 1045 & EFX68045.1 & Daphnia pulex & Partial \\
\hline DNA topoisomerase 2 like & 1808 & 988 & XP_002428978.1 & Pediculus humanus corporis & Partial \\
\hline Ubiquitin-activating enzyme like & 3834 & 966 & EFX89910.1 & Daphnia pulex & Partial \\
\hline Pre-mRNA-processing-splicing factor like & 1896 & 950 & EFX85628.1 & Daphnia pulex & Partial \\
\hline Peroxinectin & 2721 & 944 & ABB55269.2 & Fenneropenaeus chinensis & Full length \\
\hline Translation initiation factor like & 2460 & 910 & EFX65461.1 & Daphnia pulex & Partial \\
\hline Elongation factor & 1651 & 863 & ADK25705.1 & Cancer borealis & Full length \\
\hline Glucose regulated protein 78 (GRP78) & 1875 & 840 & ABM92447.1 & Fenneropenaeus chinensis & Partial \\
\hline Catalase & 3032 & 834 & ACX46120.1 & Scylla paramamosain & Partial \\
\hline Polyadenylate-binding protein 1 like isoform & 2852 & 828 & XP_003398393.1 & Bombus terrestris & Full length \\
\hline ATP-synthase subunit mitochondrial & 1710 & 827 & ADC55251.1 & Litopenaeus vannamei & Full length \\
\hline Tubulin beta-2c chain & 1406 & 823 & Q94571.1 & Homarus americanus & Full length \\
\hline
\end{tabular}

'metabolic process' (3.6\%). Other 'Biological Process' categories such as 'localization', 'multicellular organismal process' are present, but at a lower percentage. In the 'Molecular Function' category, most of the terms are grouped into the 'binding' (54.3\%) and 'catalytic activity' (33.3\%) categories, followed by 'transporter activity' (3.8\%) and 'structural molecule activity' (3.5\%). Terms such as 'enzyme regulator activity, molecular transducer activity', 'nucleic acid binding transcription factor activity' and 'protein binding transcription factor activity' are also present, but constitute a smaller proportion. The 'Cellular Component' category indicates that over 95\% ('cell part') of annotated sequences are of cellular origin. Other categories such as 'extracellular region part', 'cell junction', 'synapse and macromolecular complex' are 351 only present in small numbers.

In comparison to other studies, the distribution of genes based on 353 the GO terms and the three categories is consistent. In a study carried 354 out on the porcelain crab P. cinctipes, 'binding and catalytic activity' 355 were the most represented terms in the 'Molecular Function' category 356 (Tagmount et al., 2010). In addition, 'cellular process' was the major 357 term in 'Biological Process'. The study used different GO category levels, 358 thus the distributions are difficult to compare. However, a similar classi- 359 fication was obtained for the scallop Patinopecten yessoensis and the 360 octopus Octopus vulgaris (Hou et al., 2011; Zhang et al., 2012). Only 361

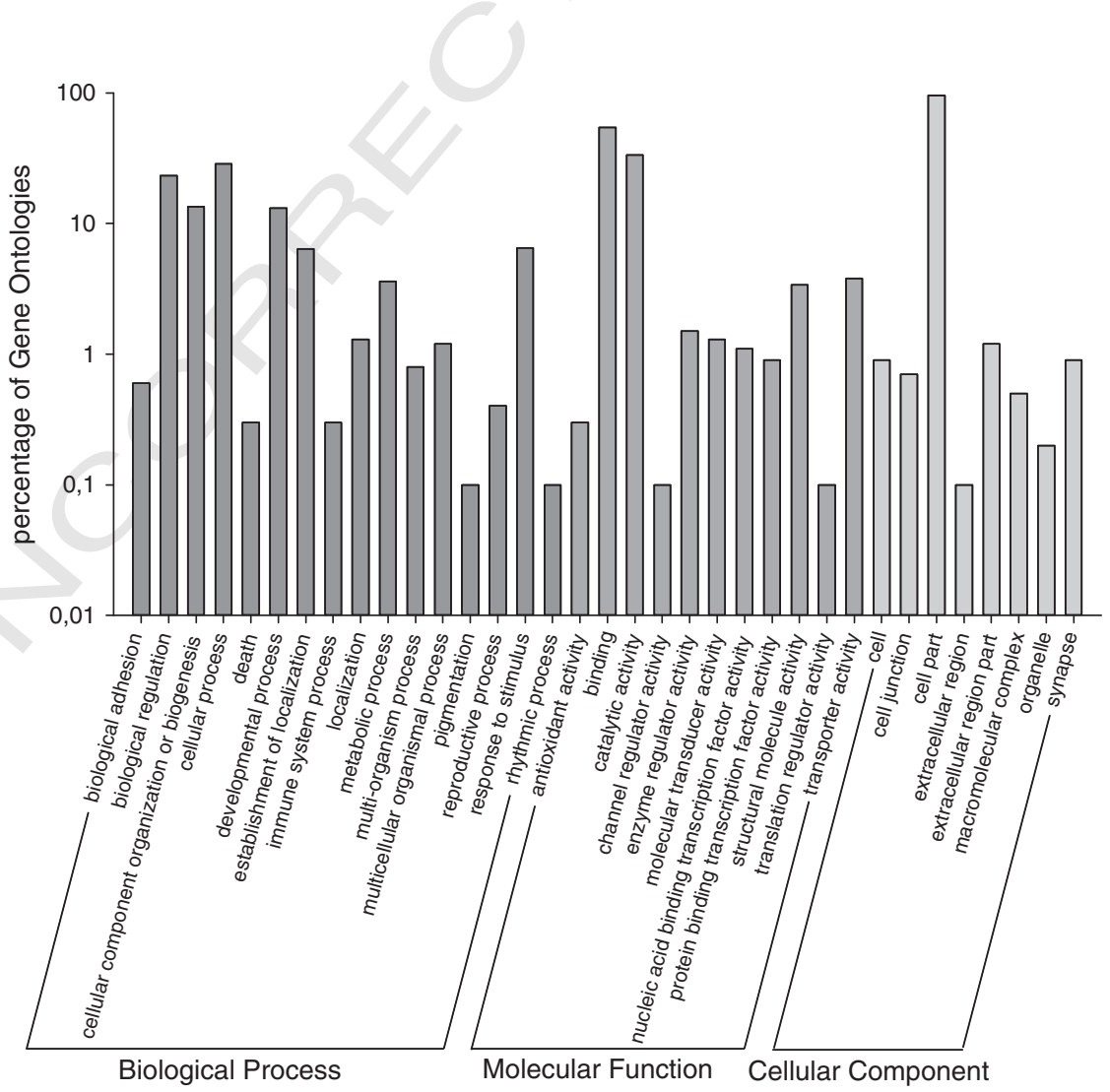

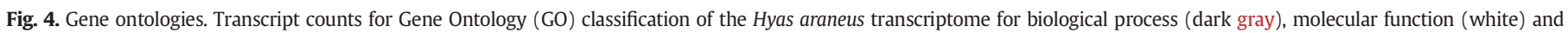
cellular component (gray) categories. 
the 'metabolic process' category seems to be underestimated in the 'Biological Process' category of $H$. araneus, as a distinctively larger proportion of 'metabolic process' GO terms (12-30\%) was observed in the former studies (Tagmount et al., 2010; Hou et al., 2011; Zhang et al., 2012). One explanation could be that a large fraction of the sequencing volume was based on gill tissue due to the focus of the sequencing project, but it could also be differences in quality and degree of sequence clustering in the assemblies. Furthermore, it must be taken into account that a possible bias exists due to the large proportion of vertebrate sequences in the common databases. However, the results of our gene ontology analysis suggest a diverse and representative gene set of the $H$. araneus transcriptome. In addition, when using the transcriptome to identify unknown proteins in a parallel proteomic study, the quality of the transcriptome was confirmed by its capacity to identify $58 \%$ of the proteins (Harms et al. unpublished).

\subsection{Comparison with $H$. americanus and P. cinctipes databases}

We used the $H$. araneus transcriptome, the porcelain crab $P$. cinctipes (Stillman et al., 2006) and the European lobster H. americanus (Towle and Smith, 2006) EST libraries for a comparative analysis to identify similarities and differences between decapod crustaceans. A tBlastx approach with an E-value cut-off of $1 \mathrm{E}^{-5}$ was performed for all sequences from each species as query against all others. The results for all sequences with a length $\geq 500 \mathrm{bp}$ are shown in Table 4 in terms of counts of best hits. For $H$. araneus, 1154 cDNAs have a hit in P. cinctipes and 1851 in $H$. americanus. The blast hits suggest a low similarity of $H$. araneus transcripts with those from the other species, with a slightly greater sequence similarity between $H$. araneus and $H$. americanus. However, considering the unequal sizes of these EST databases and the $H$. araneus transcriptome combined with a possibly different redundancy suggest that the data sets are hardly comparable by counting blast hits alone. To take the differences into account, a Markov Cluster Algorithm (MCL) clustering was applied to cluster transcripts into putative homologies. By clustering putatively related sequences into groups, the bias introduced through potentially different degrees of redundancy of transcript sequences is greatly reduced.

Counts of such overlapping clusters are a more objective statistic to compare datasets because they are less susceptible to bias when similar degrees of redundancy among data sources cannot be ensured. In the MCL clustering, 801 clusters of $H$. araneus show an overlap with $H$. americanus and 1036 clusters share sequence similarity with $P$. cinctipes (Fig. 5). In total, 1186 clusters contain sequences from all three species' libraries. This group of sequences may represent an assemblage of genes with putative core functions within decapod crustaceans. The large numbers of species-specific clusters are in agreement with the high genomic divergence as expectable from the fact that decapods comprise a set of highly diverse taxa (Martin et al., 2009). In total, for 5599 unique clusters found in $H$. araneus alone no corresponding analog could be identified in the annotation databases for the two other decapods. In comparison to the initial tBlastx analysis, the results show distinct differences. For example, the MCL cluster numbers indicate a slightly stronger similarity of $P$. cinctipes, not $H$. americanus, to $H$. araneus. All three species belong to the order of decapod crustaceans, yet differ in their classification to different infraorders ( $P$. cinctipes: Anomura; H. araneus: Brachyura; H. americanus: Astacidea). Even if the phylogenetic

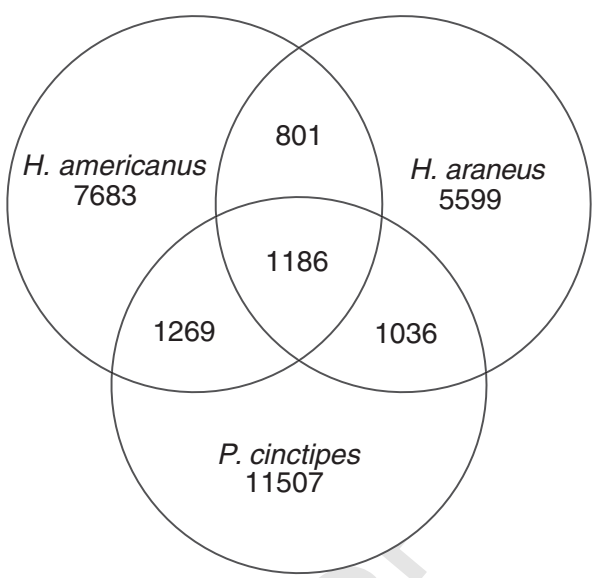

Fig. 5. Distribution of the MCL clusters built from tBlastx hits. Comparative sequence analysis of the Petrolisthes cinciptes and Homarus americanus EST libraries with the Hyas araneus transcriptome.

taxonomy is far from being completely understood, the closer relation 416 of $H$. araneus and $P$. cinctipes demonstrated by the MCL clustering is 417 supported by numerous morphological and molecular analyses. 418 Phylogenetic studies proposed an Anomura and Brachyura clade and 419 a more distant Astacidea clade (Scholtz and Richter, 1995; Ahyong 420 and O'Meally, 2004; Tsang et al., 2008). However, the approach is con- 421 sidered as an initial effective method and more comprehensive analy- 422 ses including multiple species are needed to demonstrate in how far 423 the results of the performed library clustering are taking sequencing 424 biases implicitly into consideration, and how interpretation in func- 425 tional terms can be achieved.

In terms of sequence counts, 15,111 $\mathrm{H}$. americanus ESTs (60\%), 427 43,005 P. cinctipes ESTs (57\%) and 7459 H. araneus transcripts (54\%) 428 turned out to be species-specific. In the common core of 1186 MCL- 429 clusters, corresponding sequence counts were 5009 ( $H$. americanus), 430 17,773 (P. cinctipes) and 3245 (H. araneus), respectively. It is observed 431 that the mean cluster sizes of $P$. cinctipes-specific transcript sequences 432 are significantly larger than those of $H$. araneus. This highlights that 433 the MCL-clustering leads to cluster sizes roughly proportional to the 434 size of the libraries, indicating that differences in, for example, redun- 435 dancy or sequencing depths are considered by clustering in a plausible 436 manner. Overlap estimates between transcriptomic libraries can be ef- 437 fectively computed by clustering to reduce the effects of extensive 438 amounts of transcript variants or large genome expansions. 439

The derived clustering structure can be analyzed in more detail by 440 relating the transcript sequences to a defined set of assumed universal 441 homologies. For this, we used Core Eukaryotic Genes Mapping Ap- 442 proach (CEGMA) profiles to screen transcripts for universal eukaryotic 443 functions using trpsblastn and an E-value cutoff of $1 \mathrm{E}^{-9}$ (Altschul 444 et al., 1997; Windisch et al., 2012). A total of 961 hits of H. araneus tran- 445 scripts within the CEGMA dataset including multiple hits to 377 unique 446 CEGMA profiles were found, corresponding to a 82\% CEGMA-hit cover- 447 age of the $H$. araneus library. From these, 278 were found in the MCL- 448 derived core set of transcripts, and 57 CEGMA-profiles were located in 449 the H. araneus-specific MCL clusters. This finding can be explained by 450 an inappropriate clustering and/or limited library depths resulting in 451

Table 4

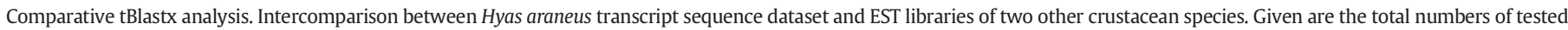
sequences for each species, the number of sequences with a blast hit in the comparative species/common core and the percentage of the respective total number of sequences.

\begin{tabular}{|c|c|c|c|c|c|}
\hline Species & $\begin{array}{l}\text { Total number } \\
\text { of sequences }\end{array}$ & $\begin{array}{l}\text { No. of blasthits in } \\
\text { H. araneus }\end{array}$ & $\begin{array}{l}\text { No. of blasthits in } \\
\text { P. cinctipes }\end{array}$ & $\begin{array}{l}\text { No. of blasthits in } \\
\text { H. americanus }\end{array}$ & $\begin{array}{l}\text { No. of blasthits in } \\
\text { the common core }\end{array}$ \\
\hline H. araneus & 13,709 & & $1154(8.4 \%)$ & 1851 (13.5\%) & 3245 (23.7\%) \\
\hline P. cinctipes & 75,298 & 7468 (9.9\%) & & 7034 (9.3\%) & $17,773(23.6 \%)$ \\
\hline H. americanus & 25,185 & $1939(7.7 \%)$ & $3126(12.4 \%)$ & & 5009 (19.9\%) \\
\hline
\end{tabular}


insufficient assemblies of the non- $H$. araneus libraries. In terms of amounts of clusters, in the core set of transcript 321 clusters contained hits to CEGMA-profiles from $H$. araneus transcripts, with 115 clusters containing more than one hit. In these clusters, 28 had hits to more than one CEGMA-profile, with a maximum of 6 different CEGMAprofiles (multiplicity 6 ). This points to limitations of the clustering approach when combined with homology information derived from partly incomplete transcriptomic sequences from non-model organisms with model organism genome databases. This is further supported by the finding that cluster-size and multiplicity of CEGMA-hits weakly correlate ( $p=0.64$, Spearman rank). It should be noted that the non$H$. araneus libraries also cover $\sim 60 \%$ of the CEGMA-profiles within the core set of clusters, confirming that the overlapping clustering contains the majority of preserved core functions.

The GO enrichment analysis of the core set of annotated sequences of $H$. araneus revealed a variety of over-represented terms from the ontologies of 'Molecular Functions', 'Biological Processes' and 'Cellular Compounds', respectively, but only 3 under-represented terms from the GO category 'Cellular Components' (see supporting information Table A). A clear picture of categories associated with the common core that could be interpreted as a representative functional clustering (e.g. with housekeeping genes dominating) within the decapod crustaceans does not become obvious. However, we observed a majority of closely interrelated terms under the GO term 'nucleotide metabolic process' within the 'Biological Process' category. This finding deserves further critical analyses with respect to the influence of assembly quality as well as of transcriptome complexity, e.g. presence of splice variants, in general.

\subsection{Special characteristic in the H. araneus transcriptome - hypothesis for} heritable, anti-viral immunity

One striking observation in the $H$. araneus transcriptome was the large amount of sequences identified as reverse transcriptase (RT). A total of 56 transcripts with a significant blast hit (E-value of $\leq 1 \mathrm{E}^{-25}$; score $\geq 150$ ) were identified as RT or RT-like sequences and thereby constitute about $0.8 \%$ of all annotated transcripts of the $H$. araneus transcriptome. RTs are used to generate cDNA and are typically found in retroviruses to integrate their RNA genomes into the host genome, resulting in a replication along with the host cell. However, sequences for RTs from retro-transposons, retro-viruses, or viral-like elements have been previously observed in the genome of insects (Terzian et al., 2001; Eickbush and Jamburuthugoda, 2008). Furthermore, the occurrence of viral and viral-like sequences in the DNA of insects and crustaceans was reported (Crochu et al., 2004; Tang and Lightner, 2006). In the genome of the black tiger prawn Penaeus monodon for example, non-infectious sequences of the Penaeus stylirostris densovirus have been found (Tang and Lightner, 2006). Based on these findings a hypothesis for a heritable, anti-viral immunity was proposed for crustaceans and insects (Flegel, 2009). According to the author, an integration of viral genome fragments into the host genome by host-derived RT and integrases (IN) could result in the generation of antisense mRNA sequences that are capable to suppress the replication of the virus. These antisense mRNAs provide protection by the RNA interference pathway, which has been already validated in shrimp (Robalino et al., 2005). Due to the variety and number of RTs of the $H$. araneus transcriptome an acute infection of the sampled animals seems unlikely, and the finding could indicate a viral recognition process similar to the pathogenassociated molecular pattern recognition system of the known innate immune system defense mechanisms of crustaceans (for review see Vasquez et al., 2009). To support the proposed viral recognition mechanism for crustaceans or at least decapod crustaceans, and concomitantly reduce the possibility that the RTs are an assemblage artifact of the $H$. araneus transcriptome as well as a contamination of viral RNA, we scanned the core-set of the MCL-clustering (see above) comprising sequences that are present in all three crustacean species' libraries for
RT sequences. We found 45 RT-transcripts (significant blasthit: E- 516 value of $\leq 1 \mathrm{E}^{-25}$; score $\geq 150$ ) of $H$. araneus in the core set $(1.4 \%)$, a 517 nearly two-fold enrichment of RTs compared to the proportion of RTs 518 of the total transcriptome (0.8\%), which suggests that the proposed 519 viral recognition mechanism is a common feature in crustaceans. For 520 $H$. americanus and $P$. cinctipes, 49 and 70 RT-sequences could be found, 521 respectively, which correspond to $0.9 \%$ and $0.4 \%$ of the sequences of 522 the core set.

Consequently, the presence of several RT-sequences in the core-set 524 of all three species makes the presence of an assemblage artifact unlike- 525 ly and reduces the possibility for a contamination, but supports the 526 presence of a viral recognition mechanism proposed for crustaceans. 527 To further test the reliability of these findings we used the previously 528 identified RT-sequences from $H$. araneus to co-locate the sequences 529 in the common fruit fly $D$. melanogaster and the purple sea urchin 530 S. purpuratus sequence libraries. D. melanogaster as a model organism 531 with a fully sequenced genome provides an excellent basis for this 532 hypothesis. It is further known that D. melanogaster comprises RT- 533 sequences as well as virus like fragments in the genome (Kim et al., 534 1994; Nefedova et al., 2011). The S. purpuratus genome was chosen as ma- 535 rine outlier. The overlap of $H$. araneus RT-sequences with $D$. melanogaster 536 revealed no RT-sequences of $D$. melanogaster, while for S. purpuratus 34537 RT-sequences could be identified. The presence of overlapping RT- 538 sequences with the $S$. purpuratus transcriptome indicates that the hy- 539 pothesis proposed for crustaceans and insects possibly can be expanded 540 to other invertebrates. The absence of homologous RT-sequences in the 541 D. melanogaster library suggests thereby that the RT-sequences found in 542 the $H$. araneus transcriptome, in the EST libraries of the other crusta- 543 ceans as well as in the sea urchin library seem to be specific for marine 544 species possibly indicating an adaptation to marine habitats and a differ- 545 ent viral composition. Several sequences, identified as integrases and 546 transposases of the $H$. araneus transcriptome further support the possi- 547 ble integration of viral fragments in the genome and thus the proposed 548 heritable, anti-viral immunity.

The present study could demonstrate the occurrence of a variety of 550 RT-sequences in different decapod crustaceans and thus support the 551 hypothesis of an integration of viral genome fragments into the host 552 genome by host-derived RT. Besides in insects and crustaceans our 553 data indicate a possible presence of a similar mechanism in other inver- 554 tebrates (sea urchin). Furthermore, the findings suggest that the identi- 555 fied RT-sequences are marine specific. Although, the presence of the 556 transcribed sequences alone is insufficient to verify the hypothesis and 557 further investigations of the genome for viral inserts are indispensible. 558 However, the several findings presented here already support the 559 hypothesis and should promote further studies.

\section{Conclusion}

In this study we characterized the transcriptome of the Arctic spider 562 $\mathrm{crab} H$. araneus. The use of normalized cDNA libraries with samples from 563 different tissues, collected after animal exposure to a variety of different 564 abiotic conditions, and a high-throughput GS FLX sequencing in combi- 565 nation with additional Illumina sequencing, resulted in high-quality 566 reads. The reads were assembled to 20,479 transcripts, $35 \%$ of them 567 were functionally annotated. Thus, the $H$. araneus transcriptomic data 568 provides a solid basement for future expression profiling and genomic 569 studies in this physiological model.

The transcripts will significantly enhance the still small amount of 571 available sequence data for crustaceans. This is even more important 572 in light of the expected high genomic diversity within the decapods, 573 requiring additional genome projects besides the Daphnia genome. The 574 proposed overlap estimates in terms of clusters of similar transcript se- 575 quences by MCL, adopted here on transcriptomic data for the first time, 576 allowed to effectively compare non-model organism transcriptomic li- 577 braries. Since we were able to determine special features and homolo- 578 gies (e.g. RTs) even in preliminary transcriptomes of crustaceans and 579 
other marine invertebrates (sea urchin), its general applicability as methodological framework has to be validated by similar questions of further organism groups.

Supplementary data to this article can be found online at http://dx. doi.org/10.1016/j.cbd.2013.09.004.

\section{Acknowledgments}

Sincere thanks go to the scientific divers of the Alfred Wegener Institute and especially Max Schwanitz for animal collection. We would further like to thank the Max Planck Institute of Molecular Genetics and Dr. Richard Reinhardt for cDNA library construction and 454 sequencing.

\section{References}

Ahyong, S.T., O'Meally, D., 2004. Phylogeny of the Decapoda Reptantia: resolution using three molecular loci and morphology. Raffles Bull. Zool. 52, 673-693.

Altschul, S.F., Madden, T.L., Schäfler, A.A., Zhang, J., Zhang, Z., Miller, W., Lipman, D.J., 1997. Gapped BLAST and PSI-Blast: a new generation of protein database search programs. Nucleic Acids Res. 25, 3389-2402.

Carson, M., Falcon, S., Pages, H., Li, N., 2010. GO.db: a set of annotation maps describing the entire gene ontology. R Package version 2.7.1.

Chevreux, B., Wetter, T.S.S., 1999. Genome sequence assembly using trace signals and additional sequence information. Comput. Sci. Biol. 99, 45-56.

Conesa, A., Gotz, S., Garcia-Gomez, J.M., Terol, J., Talon, M., Robles, M., 2005. Blast2GO: a universal tool for annotation, visualization and analysis in functional genomics research. Bioinformatics 21, 3674-3676.

Consortium, G.O., 2008. The gene ontology project in 2008. Nucleic Acids Res. 36, D440-D444.

R Core Team, 2012. R: A Language and Environment for Statistical Computing. R Foundation for Statistical Computing, Vienna, Austria.

Crochu, S., Cook, S., Attoui, H., Charrel, R.N., De Chesse, R., Belhouchet, M., Lemasson, J.J., de Micco, P., de Lamballerie, X., 2004. Sequences of flavivirus-related RNA viruses persist in DNA form integrated in the genome of Aedes spp. mosquitoes. J. Gen. Virol. 85, 1971-1980.

De Gregoris, T.B., Rupp, O., Klages, S., Knaust, F., Bekel, T., Kube, M., Burgess, J.G., Arnone, M.I., Goesmann, A., Reinhardt, R., Clare, A.S., 2011. Deep sequencing of naupliar-, cyprid- and adult-specific normalised Expressed Sequence Tag (EST) libraries of the acorn barnacle Balanus amphitrite. Biofouling 27, 367-374.

Eickbush, T.H., Jamburuthugoda, V.K., 2008. The diversity of retrotransposons and the properties of their reverse transcriptases. Virus Res. 134, 221-234.

Enright, A.J., Van Dongen, A., Ouzounis, C.A., 2002. An efficient algorithm for large-scale detection of protein families. Nucleic Acids Res. 30, 1575-1584.

Feldmeyer, B., Wheat, C.W., Krezdorn, N., Rotter, B., Pfenninger, M., 2011. Short read Illumina data for the de novo assembly of a non-model snail species transcriptome (Radix balthica, Basommatophora, Pulmonata), and a comparison of assembler performance. BMC Genomics 12, 317.

Flegel, T.W., 2009. Hypothesis for heritable, anti-viral immunity in crustaceans and insects. Biol. Direct 4, 32

Gotz, S., Garcia-Gomez, J.M., Terol, J., Williams, T.D., Nagaraj, S.H., Nueda, M.J., Robles, M., Talon, M., Dopazo, J., Conesa, A., 2008. High-throughput functional annotation and data mining with the Blast2GO suite. Nucleic Acids Res. 36, 3420-3435.

Hayward, P., Ryland, J., 1990. The Marine Fauna of the British Isles and North-West Europe: Introduction and Protozoans to Arthropods. Clarendon Press, Oxford.

Hou, R., Bao, Z., Wang, S., Su, H., Li, Y., Du, H., Hu, J., Wang, S., Hu, X., 2011. Transcriptome sequencing and de novo analysis for yesso scallop (Patinopecten yessoensis) using 454 GS FLX. PLOS ONE 6, e21560.

Kim, A., Terzian, C., Santamaria, P., Pélisson, A., Prud'Homme, N., 1994. Retroviruses in invertebrates: the gypsy retrotransposon is apparently an infectious retrovirus of Drosophila melanogaster. Proc. Natl. Acad. Sci. U. S. A. 91, 1285-1289.

Kumar, S., Blaxter, M.L., 2010. Comparing de novo assemblers for 454 transcriptome data. BMC Genomics 11, 571.
Lindquist, S., Craig, E.A., 1988. The heat-shock proteins. Annu. Rev. Genet. 22, 631-677. 638 Martin, J.A., Wang, Z., 2011. Next-generation transcriptome assembly. Nat. Rev. Genet. 12, 639 671-682.

Martin, J.W., Crandall, K.A., Felder, D.L., 2009. Decapod Crustacean Phylogenetics. CRC 641 PressI Llc.

Nefedova, L.N., Mannanova, M.M., Kim, A.I., 2011. Integration specificity of LTR- 643 retrotransposons and retroviruses in the Drosophila melanogaster genome. Virus 644 Genes 42, 297-306.

Parra, G., Bradnam, K., Korf, I., 2007. CEGMA: a pipeline to accurately annotate core genes 646 in eukaryotic genomes. Bioinformatics 23, 1061-1067. 647

Riesgo, A., Andrade, S.C., Sharma, P.P., Novo, M., Perez-Porro, A.R., Vahtera, V., Gonzalez, 648 V.L., Kawauchi, G.Y., Giribet, G., 2012. Comparative description of ten transcriptomes 649 of newly sequenced invertebrates and efficiency estimation of genomic sampling in 650 non-model taxa. Front. Zool. 9, 33.

Robalino, J., Bartlett, T. Shepard, E. Prior, S., Jaramillo, G Scura, E Chapman, RW Gross, 652 P.S., Browdy, C.L., Warr, G.W., 2005. Double-stranded RNA induces sequence-specific 653 antiviral silencing in addition to nonspecific immunity in a marine shrimp: conver- 654 gence of RNA interference and innate immunity in the invertebrate antiviral re- 655 sponse? J. Virol. 79, 13561-13571.

656

Schiffer, M., Harms, L., Pörtner, H.O., Lucassen, M., Mark, F.C., Storch, D., 2012. Tolerance of 657 Hyas araneus zoea I larvae to elevated seawater $\mathrm{PCO}_{2}$ despite elevated metabolic 658 costs. Mar. Biol.

Scholtz, G., Richter, S., 1995. Phylogenetic systematics of the Reptantian Decapoda (Crus- 660 tacea, Malacostraca). Zool. J. Linnean Soc. 113, 289-328. 661

Shi, Y., Yu, C., Gu, Z., Zhan, X., Wang, Y., Wang, A., 2013. Characterization of the pearl 662 oyster (Pinctada martensii) mantle transcriptome unravels biomineralization genes. 663 Mar. Biotechnol. 15, 175-187.

Simpson, J.T., Wong, K., Jackman, S.D., Schein, J.E., Jones, S.J., Birol, I., 2009. ABySS: a paral- 665 lel assembler for short read sequence data. Genome Res. 19,1117-1123. 666

Sperstad, S.V., Haug, T., Vasskog, T., Stensvag, K., 2009. Hyastatin, a glycine-rich multi- 667 domain antimicrobial peptide isolated from the spider crab (Hyas araneus) hemo- 668 cytes. Mol. Immunol. 46, 2604-2612. 669

Stillman, J.H., Teranishi, K.S., Tagmount, A., Lindquist, E.A., Brokstein, P.B., 2006. Construc- 670 tion and characterization of EST libraries from the porcelain crab, Petrolisthes 671 $\begin{array}{lr}\text { cinctipes. Integr. Comp. Biol. 46, 919-930. } & 672\end{array}$

Tagmount, A., Wang, M., Lindquist, E., Tanaka, Y., Teranishi, K.S., Sunagawa, S., Wong, M., 673 Stillman, J.H., 2010. The porcelain crab transcriptome and PCAD, the porcelain crab 674 microarray and sequence database. PLoS One 5, e9327. 675

Tang, K.F.J., Lightner, D.V., 2006. Infectious hypodermal and hematopoietic necrosis virus 676 (IHHNV)-related sequences in the genome of the black tiger prawn Penaeus monodon 677 from Africa and Australia. Virus Res. 118, 185-191.

Terzian, C., Pélisson, A., Bucheton, A., 2001. Evolution and phylogeny of insect endogenous 679 retroviruses. BMC Evol. Biol. 1.

Towle, D.W., Smith, C.M., 2006. Gene discovery in Carcinus maenas and Homarus 681 americanus via expressed sequence tags. Integr. Comp. Biol. 46, 912-918. 682

Tsang, L.M., Ma, K.Y., Ahyong, S.T., Chan, T.-Y., Chu, K.H., 2008. Phylogeny of Decapoda 683 using two nuclear protein-coding genes: origin and evolution of the Reptantia. 684 Anglais 48, 359-368.

Vasquez, L., Alpuche, J., Maldonado, G., Agundis, C., Pereyra-Morales, A., Zenteno, E., 2009. 686 Review: immunity mechanisms in crustaceans. Innate Immun. 15, 179-188. 687

Walther, K., Sartoris, F.-J., Bock, C., Pörtner, H.O., 2009. Impact of anthropogenic ocean 688 acidification on thermal tolerance of the spider crab Hyas araneus. Biogeosciences 6. 689

Walther, K., Anger, K., Pörtner, H.O., 2010. Effects of ocean acidification and warming on 690 the larval development of the spider crab Hyas araneus from different latitudes 691 $\left(54^{\circ}\right.$ vs. $\left.79^{\circ} \mathrm{N}\right)$. Mar. Ecol. Prog. Ser. 417, 159-170.

Wheat, C.W., 2010. Rapidly developing functional genomics in ecological model systems 693 via 454 transcriptome sequencing. Genetica 138, 433-451.

Windisch, H.S., Lucassen, M., Frickenhaus, S., 2012. Evolutionary force in confamiliar ma- 695 rine vertebrates of different temperature realms: adaptive trends in zoarcid fish 696 transcriptomes. BMC Genomics 13, 549 .

Zhang, X., Mao, Y., Huang, Z., Qu, M., Chen, J., Ding, S., Hong, J., Sun, T., 2012. Tran- 698 scriptome analysis of the Octopus vulgaris central nervous system. PLoS ONE 7, 699 e40320.

Zittier, Z.M.C., Hirse, T., Pörtner, H.-O., 2012. The synergistic effects of increasing temper- 701 ature and $\mathrm{CO}_{2}$ levels on activity capacity and acid-base balance in the spider crab, 702 Hyas araneus. Mar. Biol. xx, xxx. 